\title{
COMFORTABLE biodegradable stents in a new CENTURY?
}

\section{T} he introduction of biodegradable or bioresorbable polymer coatings is a promising development in stent technology. The investigators of two studies published in May 2014 describe follow-up data from patients who received antiplatelet-eluting biodegradable stents. The results of both studies suggest that stents with a bioresorbable polymer surface offer improved benefits over permanent polymer or bare-metal stents.

In the CENTURY II trial, the efficacy and safety of a sirolimus-eluting stent coated with a bioresorbable polymer that is designed to resorb within 3-4 months (BP-SES) were compared with an everolimus-eluting permanent polymer stent (PP-EES) over a 9 month follow-up period. The investigators enrolled 1,123 individuals with ischaemic heart disease from 58 centres in Europe, Japan, and South Korea, and randomly assigned each patient to receive either type of stent. The intention-to-treat population included 1,119 patients (562 versus 557 patients in the BP-SES and PP-EES arms, respectively). The primary end point was freedom from target-lesion failure (a composite of cardiac death, myocardial infarction, and target-lesion revascularization).

At 9-month follow-up, freedom from target-lesion failure was similar between those patients who received a BP-SES and those with a PP-EES (95.37\% versus 94.97\%; $P=0.0001)$. Similar results were observed in the per-protocol analysis.

Stent thrombosis occurred in only five patients in each group $(0.91 \%$ versus $0.91 \% ; P=0.99)$. These results indicate that the stent with bioresorbable polymer

Floris Slooff | Dreamstime.com

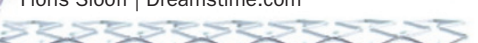

is noninferior to the everolimus-eluting permanent polymer stent.

In addition, 429 patients (214 with a BP-SES and 215 with a PP-EES) participated in an angiographic substudy at 9 months to assess lesion restenosis. In-stent late loss was greater with a BP-SES compared with a PP-EES $(0.26 \mathrm{~mm}$ versus $0.18 \mathrm{~mm} ; P=0.003)$. However, no significant difference was observed in in-stent binary restenosis between the two stent types (1.21\% with BP-SES versus $1.27 \%$ with PP-EES; $P=0.96)$.

The investigators conclude that "the new sirolimus-eluting Co-Cr stent with bioresorbable polymer ...[is] as safe and as effective as everolimus-eluting $\mathrm{Co}-\mathrm{Cr}$ stent with permanent polymer". However, these data are powered only to detect noninferiority, and a longer follow-up study is needed to investigate whether the BP-SES confers any advantage over the PP-EES.

In the COMFORTABLE AMI trial, Räber et al. compared a biolimus-eluting stent coated in a biodegradable polymer (BES) with a bare-metal stent (BMS) in 1,161 patients with ST-segment elevation myocardial infarction. Patients were randomly assigned to receive either a BES $(n=578)$ or BMS $(n=583)$, with a primary end point of any major adverse cardiovascular event (cardiac death, target-vessel reinfarction, or target-lesion revascularization) at 2 years.

The trial also included a 1-year landmark analysis, which demonstrated a benefit of BES over BMS. The incidence of the primary end point was $1.7 \%$ in those with a BES compared with 3.7\% in those with a BMS (HR 0.45, 95\% CI $0.20-1.00, P=0.049)$. Furthermore, the benefits of the BES extended beyond 1 year, despite the BES polymer being designed to degrade over 6-9 months, revealing the underlying metal stent, which in theory should have similar properties to the BMS. At 2 years, the incidence of the primary end point remained lower in patients who received a BES (5.8\% versus $11.9 \%$ with a BMS; HR 0.48, 95\% CI 0.31-0.72, $P<0.001$ ). Similarly, a composite end point of all-cause death, any reinfarction, or any revascularization was lower in patients who received a BES than in those who received a BMS (14.5\% versus $19.3 \%$; HR 0.73 , 95\% CI 0.55-0.97, $P=0.03$ ).

The COMFORTABLE AMI trial also included an angiographic substudy $(n=103)$. At 13 months, an improvement in lumen and stenosis diameter was found in patients who received a $B E S$. In-stent late loss was $0.10 \pm 0.24 \mathrm{~mm}$ with the BES compared with $0.97 \pm 0.75 \mathrm{~mm}$ with the BMS $(P<0.001)$. Binary restenosis was absent in patients who received a BES and occurred in 14 patients with a BMS $(P<0.001)$.

These results indicate that the biolimus-eluting BES is superior to a BMS in patients with ST-segment elevation myocardial infarction for preventing major adverse coronary events. However, a further study is required to assess BES safety and efficacy.

The results of both trials suggest that bioresorbable polymers can improve outcomes in patients with coronary artery disease beyond those achieved with the already widely used permanent stents. However, long-term results need to be published and assessed before a final, definitive answer can be found.

\section{Tim Geach}

Original articles Saito, S. et al. A randomized, prospective,
intercontinental evaluation of a bioresorbable polymer
sirolimus-eluting stent system: the CENTURY II (Clinical
Evaluation of New Terumo Drug-Eluting Coronary Stent
System in the Treatment of Patients with Coronary Artery
Disease) trial. Eur. Heart J. doi:10.1093/eurheartj/
ehu210 | Räber, L. et al. Biolimus-eluting stents with
biodegradable polymer versus bare-metal stents in
acute myocardial infarction: two-year clinical results of
the COMFORTABLE AMI trial. Circ. Cardiovasc. Interv.
doi:10.1161/CIRCINTERVENTIONS.113.001440

